\title{
Recommendations for Supporting Prospective Study Registration in the Basic Experimental Studies in Humans \\ And \\ Alternative Procedures for Results Dissemination
}

\author{
David Mellor and Nici Pfeiffer \\ Center for Open Science
}

\begin{abstract}
Table of Contents
Introduction

Key Principles

Stakeholder Interests

Optimization Through Balancing Trade Offs Between Stakeholders

Benefits and goals of study registration

Design Principles For Infrastructure to Meet Stakeholder Interests

Current Design and User Experience of Existing Platforms

Recommendations for Infrastructure to Meet Stakeholder Interests

Supporting Study Registration Through Journal-Funder Partnerships

Improved Outcome Reporting
\end{abstract}

\section{Introduction}

Recently, the NIH has modified the definition of clinical trial so that it applies to a much broader range of studies that previously were classified as clinical research but not specifically as trials. This definition, below, specifically includes research where behavioral outcomes are a focus of the study:

"A research study in which one or more human subjects are prospectively assigned to one or more interventions (which may include placebo or other control) to evaluate the effects of those interventions on health-related biomedical or behavioral outcomes."

With the addition of behavioral outcomes, many studies that previously would not have been "clinical trials" are now so classified. Research studies that meet both criteria for "clinical trial" and "basic research" are now referred to as basic experimental studies in humans (BESH).

The new definition that broadens the scope has created challenges on how best to meet these new standards and requirements, while still maintaining the efficiency, adaptiveness, nuance, and serendipitous opportunities that deserve to be further investigated in basic research.

These challenges are highlighted in Stage Zero/One research projects of the NIH Stage Model (https://www.nia.nih.gov/research/dbsr/nih-stage-model-behavioral-intervention-development) 
as well as in the context of certain adaptive designs or projects using the Multiphase Optimization Strategy (MOST), where optimization prior to evaluation (typically RCT) includes pilot research and identification of the most promising or feasible interventions.

The purpose of this whitepaper is to analyze and investigate ways that existing infrastructure could be improved to support these new requirements in ways that benefit all stakeholders. These stakeholders include:

- Primary investigators of BESH research

- Meta-researchers: PIs who investigate the BESH research community

- Early career researchers, often those working under the supervision of PIs

- Research participants included in BESH research studies

- Members of the public who are personally interested in the generation of evidence on research relevant to their lives

- Funders of BESH research

- Reviewers, readers, editors, and other colleagues in the scientific community who have a professional interest in the latest evidence

\section{Key Principles}

One of the underlying challenges in the shift toward more transparent and reproducible research methods, namely (pre)registration, is in designing the most efficient process for narrowing the pipeline of explanations from theoretical possibility to near assuredness. This pipeline must balance the relative risks of false positives and false negatives differently from early to later stages of research.

Early in the scientific workflow, false negatives are more detrimental, as they could lead to missing potentially important discoveries. However, the corollary to that conclusion is that one should temper any inferences derived from the conclusions of such research for anything other than follow up confirmation. Though certainty can be lowered early on, transparency cannot.

Later on, as certiny is expected to increase the impact of a finding, false positives are a larger risk because they lead to decreased confidence in scientific findings. The obvious challenge is to obtain the correct workflow and balance. There will continue to be debate about the optimum balance of credibility and discovery because of different methodologies, philosophies, and priorities by different researchers. Therefore, regardless of the philosophy of the researcher or the intended purpose of the reported results, transparency in the entire workflow is needed in order to evaluate the work.

- Key Principle 1: The guiding principle that should focus all infrastructure and policy improvement over time is transparency. 
By focusing on transparency of scientific process, infrastructure and policy makers can accommodate the diversity of methods and procedures that are present in BESH research. Study registration enables this transparency, and enables the type of rigor the confirmatory research requires when the conclusions or impact of the relevant BESH research is to be wider than purely discovery work. Later stages of research from basic science, pilot feasibility studies, observational methods, and "micro RCTs" seek to further refine the list of possible explanations to those that can be feasibly tested and defined with specific, a-priori hypotheses. It is in this transition zone, prior to more well understood, late stage clinical trials, that increased clarity is needed by the stakeholder community so as to better interpret the methods and the subsequent results.

- Key Principle 2: Infrastructure for increasing credibility must be designed for the needs of the basic research community, who can benefit from some of the rigor of late stage clinical confirmation, but are not yet expected to adhere to oversight requirements of those later stages.

While not typically labeled as "confirmatory," these BESH studies often do presume a-priori hypothesis tests and therefore are highly appropriate for preregistration. Notably, that benefit is not a function of using human study participants. Non-human animal research, and even in-vitro experiments often suffer from undisclosed flexibility in data analysis and a high number of unreported "file drawered" studies- both problems that study registration can address.

Supporting researchers in this transitional zone where BESH research flourishes requires an infrastructure that meets the needs of the ideal workflow at this stage, while providing the benefits to each of the above stakeholder groups.

- Key Principle 3: Stakeholder interests of BESH research are different than those of later stage clinical research. The infrastructure and oversight serving this community must serve those relevant interests.

\section{Stakeholder Interests}

Improving infrastructure requires an accurate understanding of stakeholder interests, including information needs, workflows, career advancement, training, and evaluation. Below is a basic overview of those who do or can interact with study registrations for $\mathrm{BESH}$ research.

Primary investigators require an efficient system that adds value to their workflow by being closely integrated into their daily routine. A registry can be a useful tool for maintaining the version of record of their most up to date research plans. These benefits can ease lab management expectations if the tools are easy to use and provide ancillary benefits. Examples of both of these include easy login, file storage and organization, version control, and collaboration. Pls also are also strongly interested in maintaining primacy of ideas (i.e. fears of 
being "scooped" are most pronounced by this stakeholder) and in maintaining the ability to publish, and are therefore most susceptible to real or perceived threats to the publishability of findings if preregistered, confirmatory findings are deemed to be less novel, significant, or exciting than unregistered analyses with increased opportunities for flexibility.

Meta-science Researchers include meta-analysts, systematic reviewers of the literature, and those researchers evaluating the process of science in order to detect shortcoming and advocate for improvements. Widespread registration of BESH research will facilitate each of these endeavors, though their interests in later discoverability of work (e.g. through lengthy checklists or forms that are used in later interface filters) can at times come into conflict with the researcher's interest in quickly creating and submitting a preregistration.

Early career researchers are also attuned to concrete incentives in the academic research community. Widely defined as graduate student through pre-tenure faculty, this community is even more highly focused on research efficiency, but with real pressure to translate that to funded grants and published papers. Those involved with conducting $\mathrm{BESH}$ research are most sensitive to signals from journals and funders. This sensitivity can be a negative force if it is applied through increased pressure or mandate compliance mechanisms. On the other hand, it can be a force for positive improvement if there is clear recognition for better practices in study registration. Infrastructure that is recognized or endorsed by key funders and journals may add to the importance of these actions.

Research participants volunteer their time and in some cases are subject to risk because of the possible benefit of scientific research to other people. Their needs and interests should likely take precedence to those of any other stakeholder. Tools that improve study design, reduce the file drawer of unreported research, and maximize the value of evidence provided by their participation need to be supported. Doing so while simultaneously protecting privacy and making data management easier (so as to minimize risk of accidental release of sensitive data, or to remove participant data when informed consent is removed) are additional considerations for infrastructure design.

Members of the public have at least two primary concerns: 1) that ongoing research that is potentially relevant to their lives is discoverable and 2) that the results of such research are disseminated with as little bias and as much credibility as possible. Any increased use of study registration increases those two goals, though particularly addressing the second goal requires additional training for reporting the results of preregistered research.

Funders want to ensure that the results of their investments lead more quickly to discoveries and more knowledge. Selectively reporting results in favor of only significant findings, or presenting the results of exploratory findings as if they were confirmatory undermine this interest. While many funders will focus more on either basic or applied science (based on their legislative or founding mandates), none benefit from biased reporting or methods that inadvertently undermine the credibility of reported findings. Preregistration benefits both 
interests by placing results into their proper context regardless of basic or applied focus. In other words, preregistration allows exploratory results to be presented as outcomes that deserve further testing and confirmatory results as having been a true confirmation.

Policy and Decision Makers BESH research, like all research, has the potential to influence decisions. Doing so, however, requires that the evidence be presented completely, without bias, and in an intuitive manner. Being able to filter by similar research questions, understanding the degree to which any given study provides evidence for a question, and access to underlying process and data help an informed reader use the presented information completely and accurately.

Colleagues in the scientific community benefit from preregistered $\mathrm{BESH}$ research by being able to more accurately evaluate asserted claims. This enables the type of scrutiny and evaluation that the "open science" community is working to achieve. Only through widespread adoption of the practice, through changing norms, increased incentives, or mandates, will such scrutiny be evenly applied to any assertion, thus leveling the playing field for all.

\section{Optimization Through Balancing Trade Offs Between Stakeholders}

Of the above stakeholders, there are three basic perspectives to take into account when designing or improving a registry system. The author when writing their registration, the author when reporting the results of their registered BESH study, and the end users or readers (colleagues, policy makers, funders, etc). The interests of the author writing their initial registration is to be as quick and efficient as possible and to help scaffold the research plan in a helpful way so as to identify any important design or analysis considerations prior to conducting the study, when such input is most likely to be useful. The researcher writing the results of their work need support and reminders to fully report each pre-specified outcome and a place to document deviations or transparent changes from the plan; as well as digital project space for relevant digital materials generated during the study process (e.g. stimuli, prompts, scripts, code, or documentation). Finally, the readers and users of these study registrations can benefit from some of the same priorities as listed before, such as clear study design and report scaffolding, but will benefit more from items that ease reuse and discovery, namely, rich meta-data that describe the type of study, the outcome categories, the evidence gathered, and other fields that allow for decision making and meta-analysis of final, reported results.

\section{Benefits and goals of study registration}


There is no single "scientific method" that can apply across any field. However, transparency in process, evidence, and methods can allow the solution most appropriate to a specific situation be applied when needed. Without transparency, the review, reader, or editor is unable to adequately evaluate the reported work. Without such transparency being normalized, it will continue to be deemed "invasive" or laced with accusations of fraud. As when a reimbursement request expects a receipt, without one assuming that they are accusing you of fraud, so should an empirical claim be accompanied by justification.

Widespread adoption of study registration for BESH research will provide the maximum amount of transparency while avoiding the unsolvable problem of proscribing a single method across vastly different methodologies. A registry can allow the user to select proscriptive design templates early on if they want to reap the benefits that those designs have on later inference; or the user can provide transparency through more open ended design specifications that allow for maximum flexibility but that constrain the later generalizability of empirical claims from the work. This cannot be achieved with a single prescriptive template but can be achieved through equal application of transparency to every study. Without that condition, those who are not incentivized, required, or expected to register will be able to avoid tough decisions until results are known and accrue the benefits of opaque practices.

\section{Design Principles For Infrastructure to Meet Stakeholder Interests}

With such a diverse group of stakeholders and a need to deliver meaningful solutions to the three key principles, the infrastructure solution will need to be well designed and engage the community of stakeholders in that process. The solution designed will need to be robust and versatile to span the needs for each stakeholder, stage of research and varied methods and study designs. While also having a low barrier to entry and efficient workflow to allow for clear articulation of methods and results for interpretation by investigators. The design must be specific to the needs of the Basic research community without passing along requirements from previous tools built for other research types.

\section{Design Principle 1}

Use of User-centric Design (UCD) methodology for developing the infrastructure to support $\mathrm{BESH}$ registrations will be critical for success in delivery of a solution the community will adopt and readily use. UCD is a process that requires explicit understanding of how each of the stakeholders will interact with the registration system, what tasks and workflows they will rely on, and what environments and tools surround those tasks that should be considered in the entire workflow. Direct engagement with the BESH research stakeholder community to conduct user research through interviews, surveys and focus groups will be the best way to ensure UCD is infused into the registration infrastructure design and development process and the needs of the stakeholder community are met by the registration process. User research should happen 
before any building begins, and throughout the entire process, especially as prototypes are produced to gather feedback from the stakeholders to inform design iterations.

\section{Design Principle 2}

"Don't make me think" is a book by Steve Krug, which has evolved into a design methodology for usable software design. The principle component is that software should be designed with concision and obviosity. In this way, less is more and keeping it simple wins for usability. Challenging the decisions made throughout the design process with Design Principle 1 where the BESH research stakeholders give feedback on usability, navigation, onboarding and learning curves on each stage ensures usability remains a focus. The continued assessment of the balance in the workflow for enough complexity to meet a range of needs for stakeholders with simple workflows with obvious actions to ensure newcomers can acclimate quickly and adopt the registration process easily into their research workflows.

\section{Design Principle 3}

User experience (UX) design is another principle that is critical in the design of the BESH research registration infrastructure, which takes an approach to information architecture that will reduce the need for special terminology. The resulting contextual language creates a pleasant, intuitive user experience where users arrive from any background to do specific tasks and their experience is meaningful and relevant, having been optimized for efficiency. BESH registration will support researchers across different domains and backgrounds who may use different and unique terminology to refer to similar concepts, which poses a challenge to that array of researchers using the same software infrastructure to complete the same registration workflow. The way the platform content is structured, along with deep understanding of the users, determines how the users will interpret the workflow. With attention paid to the information architecture to arrange the content, users will need less textual support to guide them due to the user design experience embedded into the development and delivery. But if they do need additional support to guide them through the process, the information architecture considerations will provide clear navigation of paths for successful completion of the tasks for the producers: preregistration and outcome reporting, and the consumers. This means for $\mathrm{BESH}$ investigators and stakeholders: it will be easy to navigate to take the action they came to do, for completing lengthy processes the needed steps and information are clear from the onset, choices in decision points are laid out with clarity of context, leaving and returning to where they left off is easy, missing information is identified early and often, additional help and examples are given with each question, and successful completion is the goal that each user is guided toward.

\section{Current Design and User Experience of Existing Platforms}


The goals for creating Clinicaltrials.gov as a registry for studies were to (1) register trials to reduce publication bias, (2) make ongoing and completed research discoverable, and (3) register analysis plans to distinguish between exploratory and confirmatory studies. Ultimately responding to the public outcry for transparency and accountability in the research process, particularly where human subject participants were involved in a trial study. Since coming online in 2000 CT.gov has iterated to include a wider range of trials and to require reporting of results and adverse events, having one major revamp of the platform in 2012 , with a recent request for comments in early 2020 indicating another might be in the works. Investigators are the primary users of the registry, needing to use it to comply with US public laws requiring registration of trials, who have given underwhelming reviews of the platform. Finding the platform difficult to navigate, often getting frustrated at spending lengthy sessions going in circles to complete what should be a straightforward process, and technology that hasn't kept pace with user experience design concepts or stable performance.

There have been many reviews of Clinicaltrials.gov and the $\mathrm{NIH}$ policies for registration and outcome reporting of clinical studies, suggesting there are significant gaps and a need to reimagine the registry to meet the needs of the many stakeholders. The gaps can be put into four buckets, (1) less burden on investigators entering the study details to register the study publicly, (2) higher compliance with funder requirements for reporting outcomes from studies, (3) increased access of study trials and outcomes to patients and the public, and (4) improve the rigor of submitted studies so as to improve credibility.

Customization of different workflows, with removal of irrelevant aspects in the current cilinicaltrials.gov interface will support the needs of BESH research. Specifically, removal of items such as intervention types, oversight, and contact information will serve the needs of the relevant BESH stakeholders (both researchers and later users of the research) without sacrificing the needs of patient groups (for example) who need this information in a more clinical setting. This will reduce time required to comply with funder requirements to register studies, while still meeting the most important needs of the community.

Customization of the workflows to support BESH research will further support both researchers and other stakeholders. In particular, this customization can and should include:

- More varied experimental protocol reporting. For example, specific templates for different modes of research that use existing data or that use longitudinal methods.

- Ability to report multiple measured variables, continuous predictor variables, multiple sample sizes, multiple observations, and participant assignment.

- Support for capturing experimental designs with nesting, multifactorial, within-participant designs, manipulations, correlational observations, and mixed designs.

Pairing a flexible, customizable registration workflow aimed at meeting the needs of $\mathrm{BESH}$ investigators and producing a more efficient workflow with improved outcome reporting templates supports the next gap of compliance with reporting study results. Too many trials registered on Clinicaltrials.gov never have the results reported, or are reported past the deadline 
(e.g.

https://www.sciencemag.org/news/2020/01/fda-and-nih-let-clinical-trial-sponsors-keep-results-se cret-and-break-law). Investigators recognize that reporting the results is what should be done, however, the burden on their time and financial resources is primarily resting on three hurdles (1) a maladroit user interface that lacks applicability and ease of use, (2) lack of funds to support the time to prepare results for this final leg, and (3) lack of enforcement (either formal or informal) to support a research culture where reporting is the normative behavior. Improving the user interface is possible when time is spent applying the design principles outlined above to the navigation and layout of the registry. However, attention must be paid to architect the platform to easily incorporate current and future customization requirements for supporting flexible outcome reporting appropriate for the study design. Those adjustments will minimize the burden on investigators to report their findings, and likely will mean that the time needed to do the task is much lower and keeps within the study budget. Though enforcement is needed to support the cultural shift towards transparency, along with infrastructure for BESH research registration, good UX design to make it easy and reduce burden, training and support for building communities of practice where investigators are involved in the interface design and policies, and rewarding investigators and stakeholders for complying (https://www.cos.io/blog/strategy-for-culture-change).

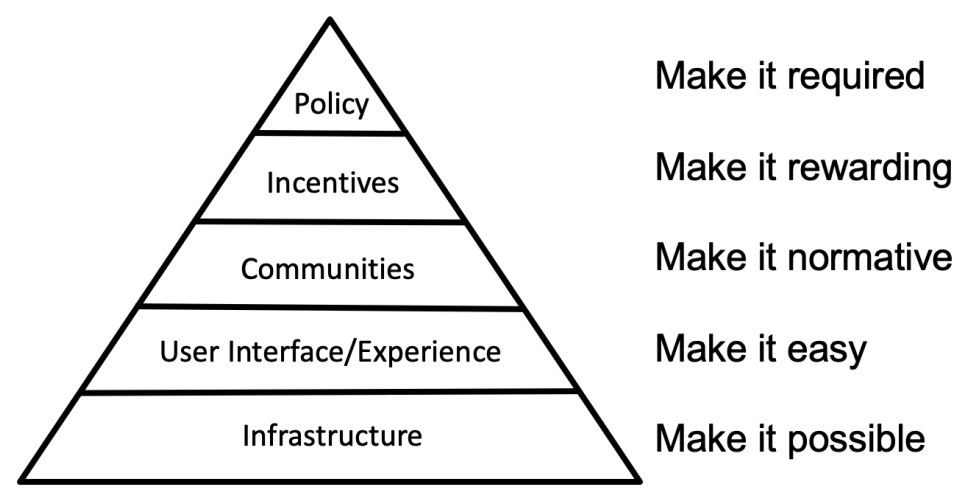

Making study trials accessible, whether for BESH or other basic research, empowers patients and the public with increased knowledge about research studies that might be recruiting patients to participate, conducting phases of the trial, or have reporting results with potential impacts to lives. Building trust and credibility between research investigators, patients and the public will mean increased support for funds enabling more innovative research resources, facilities and progress to solve our greatest health challenges. In order to increase access to study trials and outcomes to patients and the public, infrastructure and easy-to-use workflows must exist to enable investigators to report studies and their findings.

The Open Science Framework (OSF) is open source infrastructure built and maintained by the non-profit Center for Open Science (COS) which supports the entire research lifecycle and the 
collaborative management workflows for openness, transparency and reproducibility. OSF has workflows for registration of research plans, using customizable registration templates which can address the gaps with CT.gov for use by BESH investigators to register the studies. Additionally, the core OSF Registry aggregates study registrations for the general purpose solution with two external study registries (Clinitcaltrials.gov and ResearchRegistry.com) and six different registration templates (detailed in next section), as well as the ability to support communities, funders, publishers or other groups with setting up their own branded registry using the same core infrastructure. Branded registries that are launched on OSF (https://cos.io/registries) contain the core elements for registration allowing authors to choose the relevant template for the research, a linked project space on OSF with many features for managing collaboration and data sharing, a public API for programmatic access for increasing discoverability, indexing, and archival and preservation. Groups that establish a branded registry can implement a custom registration template to alleviate the gaps with the existing tools with support for the variety of BESH study designs, removal of irrelevant sections, and provide flexibility for communities to establish criteria unique to $\mathrm{BESH}$ that are incorporated into the registration template and workflow.

After registering a BESH study on an OSF Branded Registry with a custom template developed by the community of BESH investigators and stakeholders, authors can seamlessly transition into the other stages of the research lifecycle for collaborating, sharing materials (measures, protocols, intervention materials, instruments, analytic code), and reporting on the linked OSF project. Files uploaded on OSF are FAIR, with standard metadata, granular permissions and access controls for meeting a variety of workflow needs. Returning to the registration after the study completes to share the outcomes and data, is embedded within the OSF project and previous timestamped registration, reducing researcher burden and establishing efficient workflows for investigators.

\section{Recommendations for Infrastructure to Meet Stakeholder Interests}

Infrastructure can support the above interests if designed to do so. By leveraging an open source solution, stakeholders can have confidence in not being locked-in to a single solution, commercialized, or abandoned. Development of usable and relevant infrastructure depends heavily on the methodologies used in the design. We recommend incorporating the following design principles in this process: (1) User Centric Design to ensure stakeholder needs and empathy for their goals and motivations, (2) Usable software design to ensure minimal cognitive load on users and maximum usability through straightforward and uncomplicated design patterns, and (3) User Experience (UX) Design to ensure mastery of efficient and intuitive information architecture creating a pleasant experience for users and stakeholders.

Moving past the platform design into functionality and features, the infrastructure must continuously invoke stakeholder (e.g. authors and readers) empathy and goals into the 
architecture. To meet those, the infrastructure will need to be optimized to bring the flexibility required for the variety of experimental designs, meet the variety of reporting requirements and workflows, and to be built to expand appropriately for anticipated future changes. There won't be a single, one size fits all solution for all, rather a matrix of potential workflows that all lead to success for each stakeholder. A robust technological core, optimized for scaling and performance, as well as upstream and downstream integrations made possible by an open API is essential to ensure longevity of the infrastructure and contributed content. Support for communities to develop their own landing page (i.e. discovery interface) within the core platform for aggregating domain specific outputs will build communities of practice. Furthermore, these customized spaces will garner support for shifting norms toward improved transparency and accountability, while maintaining consistent $\mathrm{UI}$ and aggregation across all domains.

Applying the FAIR Principles for findability, accessibility, interoperability, and reusability are essential elements of the infrastructure. FAIR registrations will ensure that the content remains available for long term (re)use, which addresses investigator, funder, and public interests. Support for community metadata ontologies and controlled vocabularies as part of the annotation of digital objects (e.g. registrations, protocols, data, reports, analysis code, publications) ensure machine readability.

Community branded interfaces for discovering, consuming, and producing BESH registrations builds communities of practice to set standards using customizable registration templates, domain specific metadata, and custom workflows. Examples of these custom workflows include those that are available now or in the future on OSF.

Examples of registration forms that currently exist on the OSF include:

- OSF Preregistration: This is the standard preregistration form on OSF designed to be maximally relevant and exhaustive.

- Open-Ended Registration: A basic form designed to support unstructured study protocols that are uploaded by the user (for example in Word docs or PDFs).

- Registered Report Protocol Preregistration: This form is intended for those who have received an "in-principle acceptance" for a registered report from a journal.

- Preregistration Template from AsPredicted.org: This form incorporates a concise template from a third party advocate of preregistration.

- Replication Recipes: These forms are specifically designed for direct replication studies.

- Preregistration in Social Psychology.

Registration forms that are under development include:

- Studies that rely on the use of existing datasets, which are particularly useful for maximising the value of research dollars but that can be problematic if the analyst has too much insight into existing trends in the data.

- Studies that use longitudinal methods.

- Research in model development and testing.

- Studies in fMRI research. 
- Preclinical disease research.

- Conservation management studies.

- ISPOR-The Professional Society for Health Economics and Outcomes Research.

- A consensus-based registration template created by a taskforce of members from the American Psychological Association, the British Psychological Society, and the German Psychological Society.

Results reporting workflows

- Generic and domain-specific preprint servers that are connected to each OSF registration.

- A results reporting system supported by an NSF EAGER grant

There is little risk to further diversification of templates created for preregistration across multiple methods and disciplines, particularly if these registrations are created on platforms that can be connected via open APIs. This will minimize the risk of fragmentation that would otherwise exist while maximizing the potential to be inclusive across disciplines and encompass diverse communities.

\section{Supporting Study Registration Through Journal-Funder Partnerships}

One persistent "chicken and egg" problem with conducting research that is not purely exploratory, novel, or focused on creating discoveries is that it is perceived to be less exciting than confirmatory research (despite the fact that it is often reported using statistical tools design for strict a-priori hypothesis tests). This can make funding and publishing of such research more difficult. One solution to this problem is the to combine the grant review with study review process through the Registered Report format. In this format, studies are reviewed by stakeholders in both funder and publishing roles and, if approved by all parties, is granted funding for conducting the work and a promise to publish the results regardless of outcome. $\mathrm{BESH}$ research is well suited to this format because problems of under reporting, unreported flexibility in data analysis (that lead to p-hacking), and hypothesizing after results are known are present and this format is designed to mitigate them.

\section{Improved Outcome Reporting}

One emerging trend in study registration is the increasing importance of proper outcome reporting. The rules of thumb that we recommend researchers follow when reporting the results of preregistered research are:

1. Include a link to the study registration

2. Report the results of all pre-specified analysis 
3. Transparently note any added outcomes or analyses

4. Disclose any deviations or changes from the pre-specified methods and describe they possible effect on the interpretation of reported results.

While straightforward, the current system incentivizes against all four of these rules. This is partly caused by a lack of training or awareness about the issues. This lack of awareness can be addressed by clear infrastructure that enables these reports to be clearly written with sections for each of these items. Other steps that can be taken to ensure these studies are reported while following these guidelines include journal requirements that include them, funder expectations and sections in final report templates with these items and, as mentioned above, a general move toward transparency through norms, incentives, and requirements. 